\title{
Duffing-Type Oscillator with a Bounded from above Potential in the Presence of Saddle-Center Bifurcation and Singular Perturbation: Frequency Control
}

\author{
Robert Vrabel, Pavol Tanuska, Pavel Vazan, Peter Schreiber, and Vladimir Liska \\ Faculty of Materials Science and Technology, Slovak University of Technology in Bratislava, Hajdoczyho 1, 91701 Trnava, Slovakia \\ Correspondence should be addressed to Robert Vrabel; robert.vrabel@stuba.sk
}

Received 21 June 2013; Revised 9 August 2013; Accepted 21 August 2013

Academic Editor: Elena Braverman

Copyright (C) 2013 Robert Vrabel et al. This is an open access article distributed under the Creative Commons Attribution License, which permits unrestricted use, distribution, and reproduction in any medium, provided the original work is properly cited.

We analyze the dynamics of the forced singularly perturbed differential equations of Duffing's type with a potential that is bounded from above. We explain the appearance of the large frequency nonlinear oscillations of the solutions. It is shown that the frequency can be controlled by a small parameter at the highest derivative.

\section{Introduction}

Duffing's equation is regarded as one of the most important differential equations because it appears in various physical and engineering problems. For example, the periodically forced Duffing oscillator

$$
y^{\prime \prime}+\delta y^{\prime}+\alpha y+\beta y^{3}=\gamma \cos \omega t
$$

exhibits a wide variety of interesting phenomena which are fundamental to the behavior of nonlinear dynamical systems, such as regular and chaotic motions (see, e.g., [1-5] and the references therein; we also refer to the classical book of Nayfeh and Mook [6]). In this context, usually two-well potential of an unperturbed system was considered $(\beta>0$; see also [7-9]) by using analytical methods and numerical simulations.

In this case, the undamped $(\delta=0)$ and unperturbed $(\gamma=$ 0 ) Duffing's oscillator can basically exhibit two distinct types of steady-state oscillations, namely,

(i) in-well, small orbit dynamics, where the system state remains within the potential well centred at a stable equilibrium point (center); (ii) cross-well, large orbit dynamics, whose trajectories surround the three equilibrium points (saddle between two centers).

In both cases, under periodic external excitation, a chaotic motion can be observed when the control parameters are changed.

On the contrary, in this paper we consider that $\beta<0$ and a potential tends to $-\infty$ for $|y| \rightarrow \infty$, so the object can escape to infinity because of the bounded from above potential. There exist several atomic or subatomic situations in quantum physics where the total energy governing the particles contains an approximately square-well potential which is bounded from above; see, for example, [10] and discussion in [11]. For example, recently it has been found that the meson spectroscopy is better described by "confining" potential which is bounded from above; for details and references, see [10].

This paper concentrates on the mathematical aspects of systems with a potential that is bounded from above; more concretely, we focus our attention on the existence of nonlinear oscillations in the context of saddle-center bifurcation in the dynamical system describing the singularly 
perturbed forced oscillator of Duffing's type with a nonlinear restoring and a nonperiodic external driving force

$$
\epsilon^{2}\left(a^{2}(t) y^{\prime}\right)^{\prime}+f(y)=m(t), \quad 0<\epsilon \ll 1
$$

or rewriting to an equivalent set of the three first-order autonomous equations:

$$
\begin{gathered}
\epsilon y^{\prime}=\frac{w}{a(t)}, \\
\epsilon w^{\prime}=\frac{m(t)}{a(t)}-\frac{f(y)}{a(t)}-\epsilon \frac{a^{\prime}(t)}{a(t)} w, \\
t^{\prime}=1
\end{gathered}
$$

with potential with two-barrier structure. Here $a$ and $m$ are the $C^{1}$ functions on the interval $\left\langle t_{B}, t_{E}\right\rangle, a$ is positive and $f$ is a $C^{1}$ function on $\mathbb{R}$.

We show that the singular perturbation parameter $\epsilon$ play role modeling tool for the frequency control of the nonlinear oscillations arising in these systems (relationship (33)). Finally we prove that under some assumptions the solutions of (2) will rapidly oscillate, with the frequency of the oscillations increasing unboundedly as $\epsilon \rightarrow 0^{+}$.

System (3) is an example of a singularly perturbed system, because in the limit $\epsilon \rightarrow 0^{+}$, it does not reduce to a differential equation of the same type, but to an algebraicdifferential reduced system:

$$
\begin{gathered}
0=\frac{w}{a(t)}, \\
0=\frac{m(t)}{a(t)}-\frac{f(y)}{a(t)}, \\
t^{\prime}=1 .
\end{gathered}
$$

Another way to study the singular limit $\epsilon \rightarrow 0^{+}$is by introducing the new independent variable $\tau=t / \epsilon$ which transforms (3) to the system

$$
\begin{gathered}
\frac{d y}{d \tau}=\frac{w}{a(t)}, \\
\frac{d w}{d \tau}=\frac{m(t)}{a(t)}-\frac{f(y)}{a(t)}-\epsilon \frac{a^{\prime}(t)}{a(t)} w, \\
\frac{d t}{d \tau}=\epsilon .
\end{gathered}
$$

Taking the limit $\epsilon \rightarrow 0^{+}$, we obtain the so-called associated system $([12])$

$$
\begin{gathered}
\frac{d y}{d \tau}=\frac{w}{a(t)}, \\
\frac{d w}{d \tau}=\frac{m(t)}{a(t)}-\frac{f(y)}{a(t)}, \\
\frac{d t}{d \tau}=0 ; \quad \text { that is, } t=t^{*}=\text { constant, }
\end{gathered}
$$

in which $t$ plays the role of a parameter.

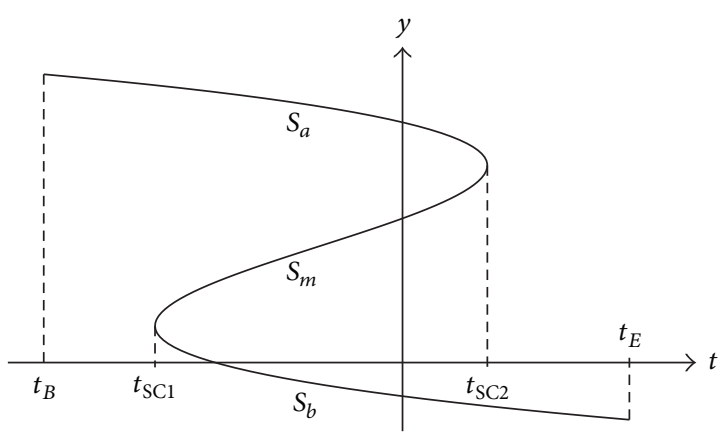

FIgURE 1: The critical manifold $S$.

Both scalings agree on the level of phase space structure when $\epsilon \neq 0$ but offer very different perspectives since they differ radically in the limit when $\epsilon=0$. The main goal of singular perturbation theory is to use these limits to understand structure in the full system when $\epsilon \neq 0$.

The critical manifold $S$ is defined as a solution of the reduced system; that is,

$$
S:=\left\{(t, y, w): t \in\left\langle t_{B}, t_{E}\right\rangle, f(y)=m(t), w=0\right\}
$$

which corresponds to a set of equilibria for the associated system (6), (7), and (8).

\section{Saddle-Center Bifurcations of Associated System}

We assume the following.

(A1) The critical manifold is S-shaped curve with two folds; that is, it can be written in the form $t=\varphi(y), t \in$ $\left\langle t_{B}, t_{E}\right\rangle$, and the function $\varphi$ has precisely two critical points, one nondegenerate minimum $y_{\mathrm{SC} 1}$ and one nondegenerate maximum $y_{\mathrm{SC} 2}$; let $y_{\mathrm{SC} 1}<y_{\mathrm{SC} 2}$. Thus, the critical manifold can be broken up into three pieces $S_{b}, S_{m}$, and $S_{a}$, separated by the minimum and maximum (Figure 1). These three pieces are defined as follows:

$$
\begin{gathered}
S_{b}=\left\{(y, \varphi(y), 0): y<y_{\mathrm{SC} 1}\right\}, \\
S_{m}=\left\{(y, \varphi(y), 0): y_{\mathrm{SC} 1}<y<y_{\mathrm{SC} 2}\right\}, \\
S_{a}=\left\{(y, \varphi(y), 0): y_{\mathrm{SC} 2}<y\right\} .
\end{gathered}
$$

(A2) Consider $\varphi^{\prime}(y) \neq 0$ for $y \neq y_{\mathrm{SC} 1}, y_{\mathrm{SC} 2}$.

(A3) Consider $(d f / d y)(y)>0$ for every $(t, y, 0) \in S_{m}$ and $(d f / d y)(y)<0$ for every $(t, y, 0) \in S_{a} \cup S_{b}$.

Due to the assumption (A3), the situation considered here substantially differs from the situation in [13], where two pieces of critical manifold, namely, $S_{a}$ and $S_{b}$, are not normally hyperbolic, and large orbit oscillations that encircle all the three pieces of critical manifold were studied (for the definition of a normal hyperbolicity of critical manifold see, e.g., [12]). In this paper, the pieces $S_{a}$ and $S_{b}$ of the critical 
manifold $S$ are normally hyperbolic, and thus the system under consideration allows another type of nonlinear oscillations, namely, small orbit oscillations around the middle piece $S_{m}$ of critical manifold $S$. For comparison, see Figure 3(a) and Figure 5.

$$
\begin{aligned}
& \text { Let } t_{\mathrm{SC} 1}=\varphi\left(y_{\mathrm{SC} 1}\right), t_{\mathrm{SC} 2}=\varphi\left(y_{\mathrm{SC} 2}\right) . \text { Denote by } \\
& u_{1}(t)=\varphi^{-1}(t): \quad t \in\left\langle t_{B}, t_{\mathrm{SC} 2}\right\rangle, y_{\mathrm{SC} 2} \leq u_{1}(t), \\
& u_{2}(t)=\varphi^{-1}(t): \quad t \in\left\langle t_{\mathrm{SC} 1}, t_{\mathrm{SC} 2}\right\rangle, y_{\mathrm{SC} 1} \leq u_{2}(t) \leq y_{\mathrm{SC} 2}, \\
& u_{3}(t)=\varphi^{-1}(t): \quad t \in\left\langle t_{\mathrm{SC} 1}, t_{E}\right\rangle, u_{3}(t) \leq y_{\mathrm{SC} 1} .
\end{aligned}
$$

The equations

$$
f(y)=m(t), \quad w=0
$$

have three solutions for $y, w$ if $t^{*} \in\left(t_{\mathrm{SC} 1}, t_{\mathrm{SC} 2}\right)$ and one if $t^{*} \in\left\langle t_{B}, t_{\mathrm{SC} 1}\right)$ and $t^{*} \in\left(t_{\mathrm{SC} 2}, t_{E}\right\rangle$. Thus the associated system (6), (7), $t=t^{*}=$ constant has three equilibria (two saddles, one center) for $t^{*} \in\left\langle t_{B}, t_{\mathrm{SC} 1}\right)$ and one equilibrium (saddle) for $t^{*} \in\left\langle t_{B}, t_{\mathrm{SC} 1}\right)$ and $t^{*} \in\left(t_{\mathrm{SC} 2}, t_{E}\right\rangle$; the eigenvalues of the jacobian are

$$
\lambda_{1,2}\left(t^{*}, y, w\right)= \pm a^{-1}\left(t^{*}\right) \sqrt{-\frac{d f}{d y}(y)}
$$

Thus, the pieces $S_{a}$ and $S_{b}$ of critical manifold $S$ are the normally hyperbolic manifolds. The points $\left(t_{\mathrm{SC} 1}, y_{\mathrm{SC} 1}, 0\right)$ and $\left(t_{\mathrm{SC} 2}, y_{\mathrm{SC} 2}, 0\right)$ of $S$ are the cusps of the codimension two ([14, $15])$, and the corresponding bifurcation is known as saddlecenter bifurcation ([16]) (Figure 2).

More precisely, at the point $t^{*}=t_{\mathrm{SC} 1}$, the birth of the saddle and the center occurs. At $t^{*}=t_{\mathrm{SC} 2}$, the left side center and the saddle coalesce. There is a unique $t_{0}^{*} \epsilon$ $\left(t_{\mathrm{SC} 1}, t_{\mathrm{SC} 2}\right)$, such that between hyperbolic points (saddles) there is a heteroclinic connection. The homoclinic loop of one hyperbolic point surrounds the corresponding elliptic (center) one for every $t^{*} \in\left(t_{\mathrm{SC} 1}, t_{\mathrm{SC} 2}\right), t^{*} \neq t_{0}^{*}$.

We use the level surfaces $H^{\epsilon}(t, y, w)=H^{\epsilon}(t)$ of the energy function $H^{\epsilon}$,

$$
\begin{aligned}
& H^{\epsilon}(t, y, w)=\frac{1}{2} w^{2}+V(t, y), \\
& V(t, y)=\int_{0}^{y} f(s) d s-m(t) y,
\end{aligned}
$$

to characterize the trajectories of (3). These surfaces in $(t, y, w)$-space are defined by

$$
w= \pm\left(2\left(H^{\epsilon}(t)-V(t, y)\right)\right)^{1 / 2}
$$

extending it as long as $w$ remains real. On the intervals $\left\langle t_{B}, t_{\mathrm{SC} 1}\right)$ and $\left(t_{\mathrm{SC} 2}, t_{E}\right\rangle$, there is a motion across a single potential barrier, and on the interval $\left(t_{\mathrm{SC} 1}, t_{\mathrm{SC} 2}\right)$, there is double barrier with a well in between.
The derivative of $H^{\epsilon}(t)$ along any solution path of (3) is

$$
\begin{aligned}
H^{\epsilon^{\prime}}(t)= & w^{\epsilon} w^{\epsilon^{\prime}}+f\left(y^{\epsilon}\right) y^{\epsilon^{\prime}}-\left[m(t) y^{\epsilon}\right]^{\prime} \\
= & w^{\epsilon}\left[-\frac{f\left(y^{\epsilon}\right)}{\epsilon a}+\frac{m(t)}{\epsilon a}-\frac{a^{\prime}}{a} w^{\epsilon}\right] \\
& +f\left(y^{\epsilon}\right) y^{\epsilon^{\prime}}-\left[m(t) y^{\epsilon}\right]^{\prime} \\
= & -\frac{a^{\prime}(t)}{a(t)}\left(w^{\epsilon}\right)^{2}-m^{\prime}(t) y^{\epsilon} .
\end{aligned}
$$

The main objective of this paper is to prove the strongly nonlinear oscillations on the interval $\left(t_{\mathrm{SC} 1}, t_{\mathrm{SC} 2}\right)$ as possible scenario of behavior of the solutions for problem (2). For this reason, we rewrite the differential equation (2) in the system form

$$
\begin{aligned}
y^{\prime} & =\frac{w}{\epsilon a(t)}, \\
(a(t) w)^{\prime} & =\frac{m(t)}{\epsilon}-\frac{f(y)}{\epsilon} .
\end{aligned}
$$

Then, we make a change of variables from rectangular coordinates $(y, w)$ to the dynamic polar coordinates $(r, \gamma)$ centered at $\left(u_{2}(t), 0\right)$ defined by the equations

$$
y=u_{2}(t)+r \cos \gamma, \quad a(t) w=-r \sin \gamma,
$$

and let us consider the system (17) on the interval $\left(t_{\mathrm{SC} 1}, t_{\mathrm{SC} 2}\right)$ in these new coordinates. The function $u_{2}(t)$ acting in the first polar transform equation corresponds to the middle piece $S_{m}$ of critical manifold $S$. At first, we derive the differential equation for polar angle $\gamma$, which is crucial for an analysis of nonlinear oscillations in this system. Dividing formally the second transform equation by the first, we get

$$
\tan \gamma=-\frac{a w}{y-u_{2}}
$$

Differentiating this equation with respect to $t$, we consecutively have

$$
\frac{1}{\cos ^{2} \gamma} \gamma^{\prime}=-\left[\frac{a w}{y-u_{2}}\right]^{\prime}=\frac{a w y^{\prime}}{\left(y-u_{2}\right)^{2}}-\frac{(a w)^{\prime}}{y-u_{2}}-\frac{a w u_{2}^{\prime}}{\left(y-u_{2}\right)^{2}} .
$$

Finally, using (17) and (18), we obtain the following differential equation for $\gamma$ :

$$
\gamma^{\prime}=\frac{1}{\epsilon}\left[\frac{1}{a^{2}(t)} \sin ^{2} \gamma+\bar{f}(t, y) \cos ^{2} \gamma+\frac{\epsilon u_{2}^{\prime}(t)}{r^{\epsilon}(t)} \sin \gamma\right],
$$

where the radius is as follows:

$$
\begin{gathered}
r^{\epsilon}(t)=\sqrt{\left(y-u_{2}\right)^{2}+(a(t) w)^{2}}, \\
\bar{f}(t, y)=\frac{f(y)-m(t)}{y-u_{2}(t)}, \\
\bar{f}\left(t, u_{2}(t)\right) \stackrel{\text { def }}{=} \lim _{y \rightarrow u_{2}} \frac{f(y)-m(t)}{y-u_{2}(t)}=\frac{d f}{d y}\left(u_{2}(t)\right) .
\end{gathered}
$$




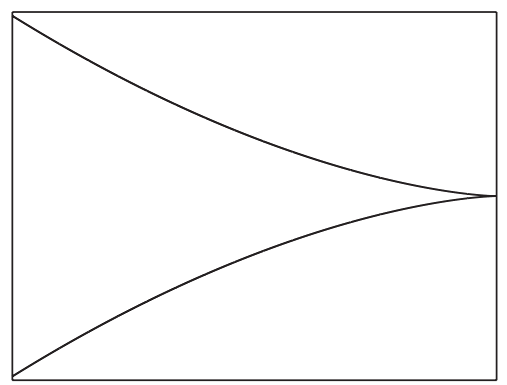

(a)

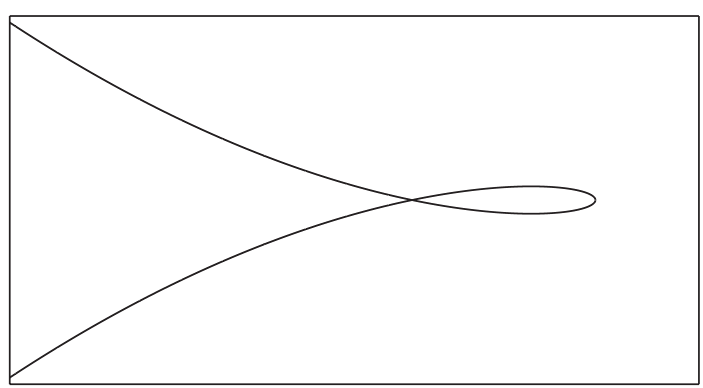

(b)

FIGURE 2: Creation and extinction (in mirror mode) of a separatrix loop in the saddle-center bifurcation at $t^{*}=t_{\mathrm{SC} 1}$ and $t^{*}=t_{\mathrm{SC} 2}$, respectively.

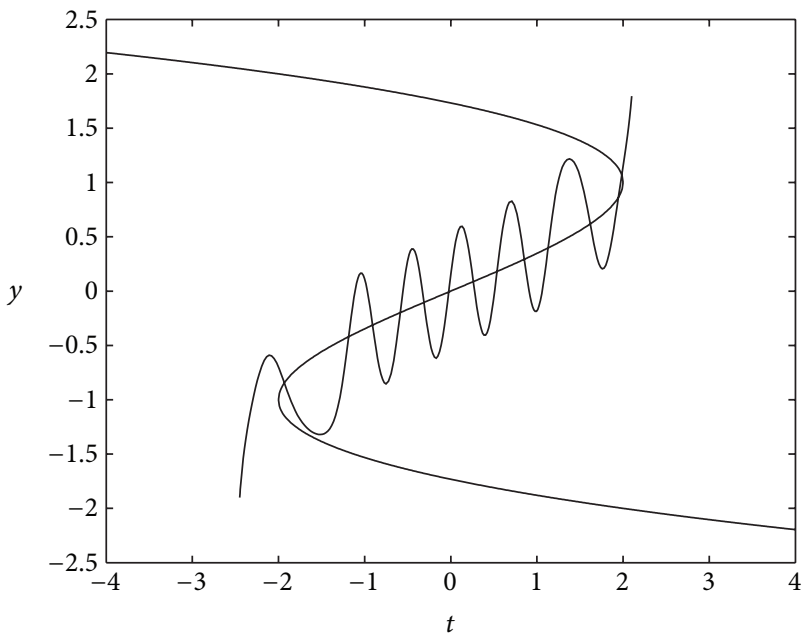

(a)

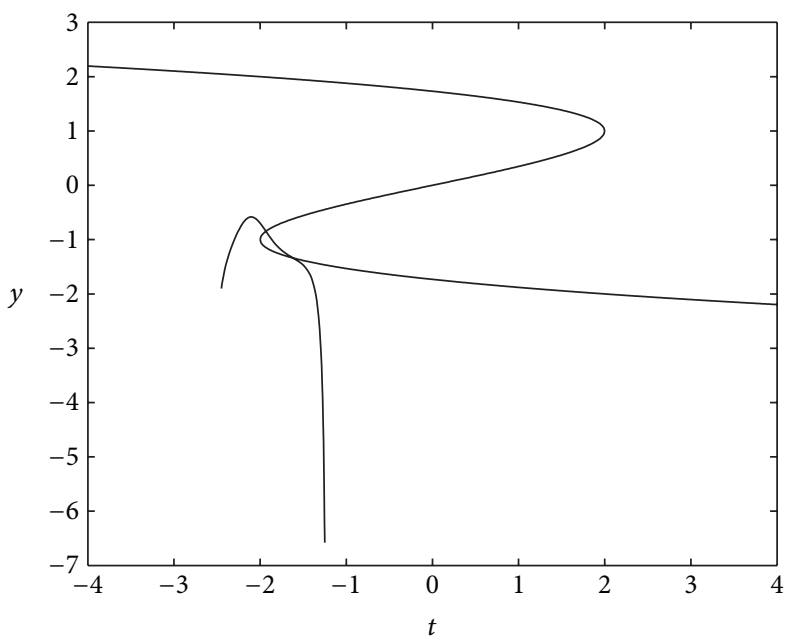

(b)

FIGURE 3: Solution of (27), (28), $\epsilon^{2}=0.0224$ on $\langle-2.45,2.1\rangle$ (a); solution of (27), (28), $\epsilon^{2}=0.0225$ on $\langle-2.45,-1.25\rangle$ (b).

Now let $K$ be a compact subset of $\left(t_{\mathrm{SC} 1}, t_{\mathrm{SC} 2}\right)$. On the periodic orbits (for fixed $t$ ), we define the minimal radius:

$$
\begin{aligned}
r_{\min }^{\epsilon}(K) \stackrel{\text { def }}{=} \min _{t \in K} r^{\epsilon}(t) & \\
=\min _{t \in K}\{ & u_{2}(t)-y_{L}^{\epsilon}(t), y_{R}^{\epsilon}(t)-u_{2}(t), \\
& \left.\sqrt{2} a(t) \sqrt{H^{\epsilon}(t)-V\left(t, u_{2}(t)\right)}\right\},
\end{aligned}
$$

where $y_{L}^{\epsilon}(t)$ and $y_{R}^{\epsilon}(t)\left(y_{L}^{\epsilon}<y_{R}^{\epsilon}\right)$ are the solutions of the equation

$$
H^{\epsilon}(t)-V(t, y)=0, \quad t \in\left(t_{\mathrm{SC} 1}, t_{\mathrm{SC} 2}\right)
$$

lying on the periodic orbit.

Obviously, $y_{i}^{\epsilon}(t) \rightarrow u_{2}\left(t_{\mathrm{SC} 1}\right)$ for $t \rightarrow t_{\mathrm{SC} 1}^{+}$and $y_{i}^{\epsilon}(t) \rightarrow$ $u_{2}\left(t_{\mathrm{SC} 2}\right)$ for $t \rightarrow t_{\mathrm{SC} 2}^{-}, i=L, R$.

Let $\delta(t)$ be a positive function such that

$$
\delta(t)+V\left(t, u_{2}(t)\right)<\min \left\{V\left(t, u_{1}(t)\right), V\left(t, u_{3}(t)\right)\right\}
$$

on $K$.

Now we make the following additional assumption.
(A4) The total energy $H^{\epsilon}(t)$ of motion described by (2) satisfies

$$
\begin{aligned}
\delta(t)+V\left(t, u_{2}(t)\right) & \leq H^{\epsilon}(t) \\
& <\min \left\{V\left(t, u_{1}(t)\right), V\left(t, u_{3}(t)\right)\right\}
\end{aligned}
$$

on a compact subset $K$ of $\left(t_{\mathrm{SC} 1}, t_{\mathrm{SC} 2}\right)$.

If a total energy of motion described by (2) satisfies the assumption (A4) on every compact subset $K$ of $\left(t_{\mathrm{SC} 1}, t_{\mathrm{SC} 2}\right)$, then $y_{i}^{\epsilon}(t) \rightarrow u_{2}\left(t_{\mathrm{SC} 1}\right)$ for $t \rightarrow t_{\mathrm{SC} 1}^{+}, t \in K$ and $y_{i}^{\epsilon}(t) \rightarrow$ $u_{2}\left(t_{\mathrm{SC} 2}\right)$ for $t \rightarrow t_{\mathrm{SC} 2}^{-}, t \in K$, and $i=L, R$.

We precede the main result on the existence of nonlinear oscillations of the solutions for (2) on the interval $\left(t_{\mathrm{SC} 1}, t_{\mathrm{SC} 2}\right)$ by important example.

Example 1. Consider Duffing's oscillator with linear excitation

$$
\epsilon^{2} y^{\prime \prime}+3 y-y^{3}=t
$$

for $\epsilon^{2}=0.0224$ subject to the initial conditions

$$
y^{\epsilon}(-2.45)=-1.9, \quad y^{\epsilon^{\prime}}(-2.45)=11.16
$$

on the interval $\langle-2.45,2.1\rangle$. 


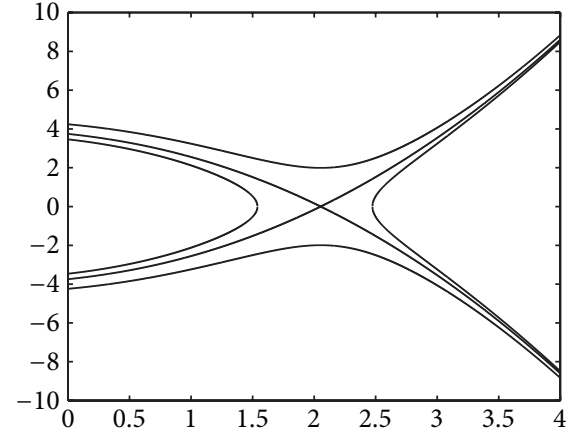

(a)

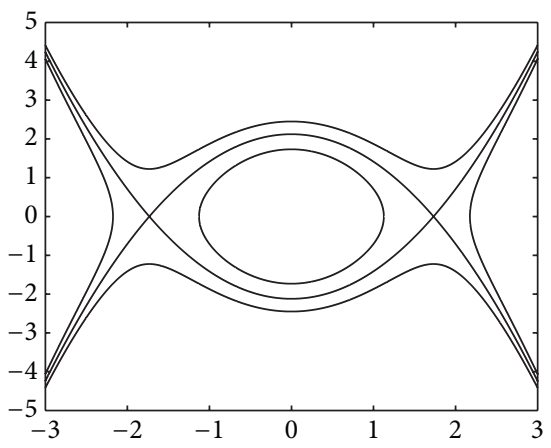

(d)

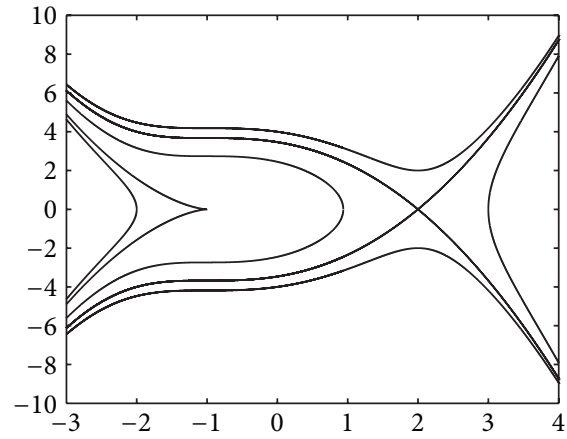

(b)

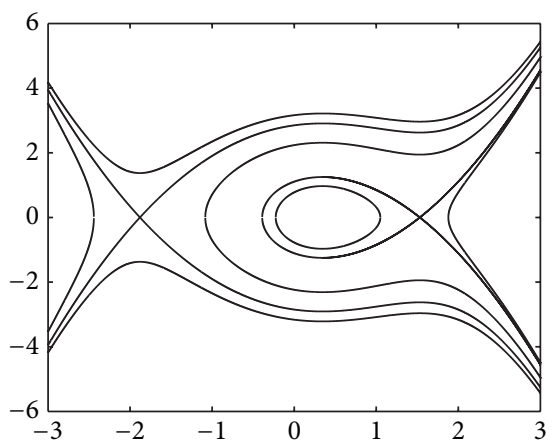

(e)

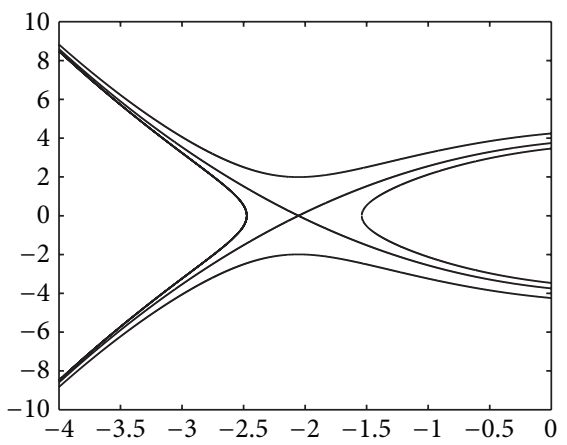

(g)

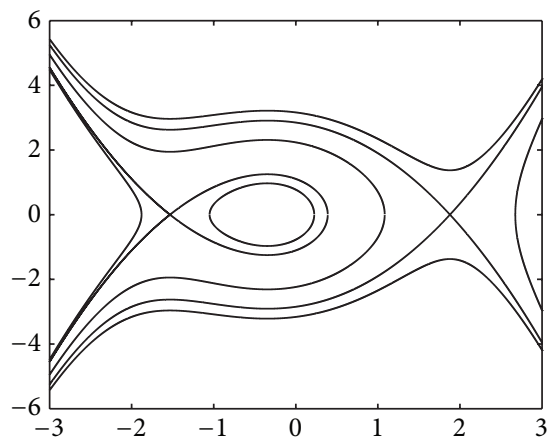

(c)

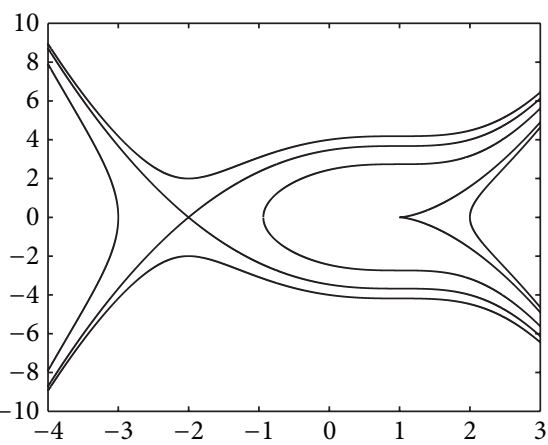

(f)

FIGURE 4: Phase portraits for $t=$ (from top-left to bottom-right): $-2.5\left(t<t_{\mathrm{SC} 1}\right) ;-2\left(t=t_{\mathrm{SC} 1}\right) ;-1\left(t_{\mathrm{SC} 1}<t<t_{\mathrm{SC} 2}\right)$; 0 (heteroclinic connections between two saddles of associated system, $\left.t_{0}^{*}=0\right) ; 1 ; 2\left(t=t_{\mathrm{SC} 2}\right) ; 2.5\left(t>t_{\mathrm{SC} 2}\right)$.

In our case $t_{B}=-2.45, t_{E}=2.1, a \equiv 1, f(y)=$ $3 y-y^{3}, m(t)=t, t_{\mathrm{SC} 1}=-2, t_{\mathrm{SC} 2}=2, y_{\mathrm{SC} 1}=-1, y_{\mathrm{SC} 2}=1$, and it is not difficult to check that the assumptions (A1)(A3) hold. Numerical results obtained from (27) subject to the initial conditions (28) using the software package MATLAB 7 are shown in Figure 3(a). These oscillations are very sensitive on the value of singular perturbation parameter $\epsilon$. Figure 3(b) shows the solution of (27), (28) for $\epsilon^{2}=0.0225$.

In order to facilitate the understanding of the qualitative behaviors of this dynamical system, we draw the $(y, w)$ phase portraits at the relevant fixed value of time $t$ (Figure 4). For comparison, Figure 5 shows the solution of initial value problem

$$
\begin{gathered}
\epsilon^{2} y^{\prime \prime}-3 y+y^{3}=-t, \\
y^{\epsilon}(-4)=3.1821, \quad y^{\epsilon^{\prime}}(-4)=0
\end{gathered}
$$

with twin-( or single-) well potential for $\epsilon^{2}=0.00354$ on the interval $\left\langle t_{B}, t_{E}\right\rangle=\langle-4,4\rangle$. This type of problems has been studied in [13].

Now we will analyze the solution of (27), (28) after the time $t_{\mathrm{SC} 2}$. The total energy (14) for (27) is

$$
H^{\epsilon}(t, y, w)=\frac{1}{2} w^{2}+\frac{3}{2} y^{2}-\frac{1}{4} y^{4}+t y,
$$

and for its derivative along the solution given by (16), we obtain

$$
H^{\epsilon^{\prime}}(t)=-y^{\epsilon}(t) .
$$

Due to the oscillations around $u_{2}(t)$ in left neighborhood of $t_{\mathrm{SC} 2}$ between $u_{1}(t)$ and $u_{3}(t)$, that is, $y^{\epsilon}>0$, the total energy decreases (dissipation) in right neighborhood of $t_{\mathrm{SC} 2}$,

$$
H^{\epsilon^{\prime}}(t)=-y^{\epsilon}(t)<0 .
$$




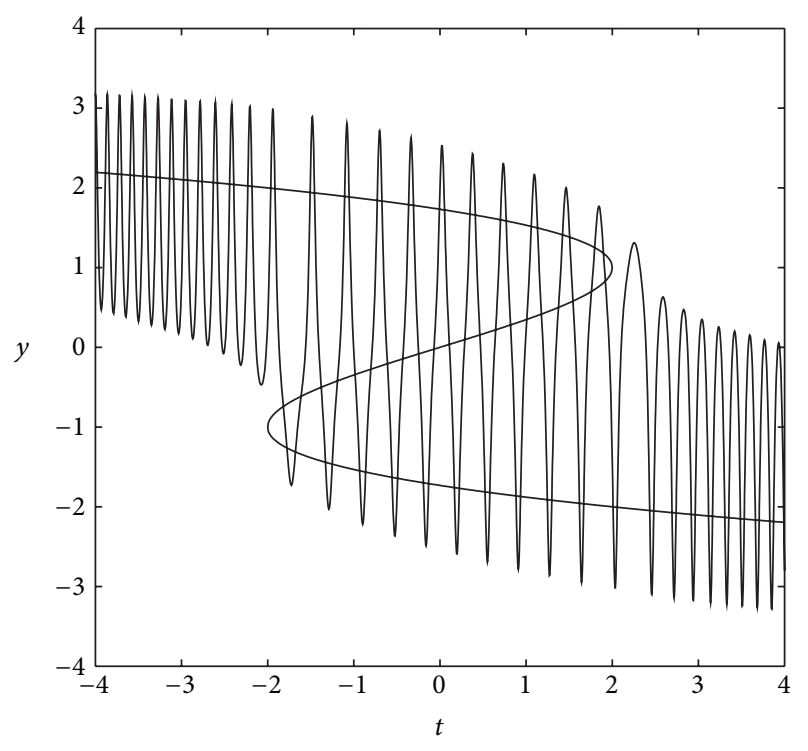

FIGURE 5: Solution of (29), $\epsilon^{2}=0.00354$ on $\langle-4,4\rangle$.

As it follows from the shape of potential $V$ and the phase portrait for $t>t_{\mathrm{SC} 2}, y^{\epsilon}\left(t^{*}\right) \rightarrow \infty$ for $\epsilon \rightarrow 0^{+}$and $t^{*} \epsilon$ $\left(t_{\mathrm{SC} 2}, t_{E}\right\rangle$.

\section{Analysis of Solutions Lying on the Periodic Orbits: Main Result}

Now we formulate the theorem on nonlinear oscillations of solutions of (2) for the motion with total energy $H^{\epsilon}(t)$ satisfying the assumption (A4). Moreover, we show that the parameter $\epsilon$ plays role modeling tool for the frequency control of the nonlinear oscillations.

Denote by $s_{\epsilon}$ the spacing between two successive zero numbers of the function $y^{\epsilon}-u_{2}$ on $K$, where $y^{\epsilon}$ is a solution of (2).

Theorem 2. Under the assumptions (A1)-(A4), the solutions of problem (2) oscillate on the compact set $K, K \subset\left(t_{S C 1}, t_{S C 2}\right)$ between $u_{1}(t)$ and $u_{3}(t)$ and

$$
\epsilon \frac{\pi}{\mu_{2}\left(K, \epsilon_{0}\right)} \leq s_{\epsilon} \leq \epsilon \frac{\pi}{\mu_{1}\left(K, \epsilon_{0}\right)}, \quad \epsilon \in\left(0, \epsilon_{0}\right],
$$

where $\mu_{1}\left(K, \epsilon_{0}\right)$ and $\mu_{2}\left(K, \epsilon_{0}\right)$ are the positive constants independent of the singular perturbation parameter $\epsilon, \epsilon \in\left(0, \epsilon_{0}\right]$.

Proof. To obtain the oscillations and the estimate of their frequencies, we analyze the differential equation (21); that is,

$$
\gamma^{\prime}=\frac{1}{\epsilon}\left[\frac{1}{a^{2}(t)} \sin ^{2} \gamma+\bar{f}(t, y) \cos ^{2} \gamma+\frac{\epsilon u_{2}^{\prime}(t)}{r^{\epsilon}(t)} \sin \gamma\right] .
$$

Taking into consideration the fact that $r_{\min }^{\epsilon}(K) \geq \Delta>0$ independently of parameter $\epsilon$ due to the assumption (A4), we can estimate that

$$
\left|\frac{\epsilon u_{2}^{\prime}(t)}{r^{\epsilon}(t)} \sin \gamma\right| \leq \epsilon \frac{\left|u_{2}^{\prime}(t)\right|}{r_{\min }^{\epsilon}(K)} \leq \epsilon\left|u_{2}^{\prime}(t)\right| \Delta \longrightarrow 0^{+}
$$

for $\epsilon \rightarrow 0^{+}$. Further for $t \in K$ and $y \in\left(u_{3}(t), u_{1}(t)\right)$, we have $\bar{f}(t, y)>0$. Thus for sufficiently small values of the singular perturbation parameter $\epsilon$, say, $\epsilon \in\left(0, \epsilon_{0}\right]$, there exist the positive constants $\mu_{1}\left(K, \epsilon_{0}\right), \mu_{2}\left(K, \epsilon_{0}\right)$, and $\mu_{1}\left(K, \epsilon_{0}\right)<$ $\mu_{2}\left(K, \epsilon_{0}\right)$ :

$$
\begin{array}{r}
\mu_{1}\left(K, \epsilon_{0}\right) \\
=\min \left[\frac{1}{a^{2}(t)} \sin ^{2} \gamma+\bar{f}(t, y) \cos ^{2} \gamma+\frac{\epsilon_{0} u_{2}^{\prime}(t)}{r^{\epsilon}(t)} \sin \gamma ;\right. \\
\left.t \in K, y \in\left\langle y_{L}^{0}(t), y_{R}^{0}(t)\right\rangle, \gamma \in\langle 0,2 \pi\rangle\right], \\
\mu_{2}\left(K, \epsilon_{0}\right) \\
=\max \left[\frac{1}{a^{2}(t)} \sin ^{2} \gamma+\bar{f}(t, y) \cos ^{2} \gamma+\frac{\epsilon_{0} u_{2}^{\prime}(t)}{r^{\epsilon}(t)} \sin \gamma ;\right. \\
\left.t \in K, y \in\left\langle y_{L}^{0}(t), y_{R}^{0}(t)\right\rangle, \gamma \in\langle 0,2 \pi\rangle\right],
\end{array}
$$

where $y_{L}^{0}(t)$, and $y_{R}^{0}(t)$ are the roots of the equation

$$
\min \left\{V\left(t, u_{1}(t)\right), V\left(t, u_{3}(t)\right)\right\}-V(t, y)=0,
$$

lying on the periodic orbit.

Putting (35) into the definitions of constants $\mu_{1}\left(K, \epsilon_{0}\right)$, and $\mu_{2}\left(K, \epsilon_{0}\right)$, we obtain that

$$
\begin{aligned}
& \mu_{1}\left(K, \epsilon_{0}\right) \\
& \geq \min \left[\frac{1}{a^{2}(t)} \sin ^{2} \gamma+\bar{f}(t, y) \cos ^{2} \gamma-\epsilon_{0}\left|u_{2}^{\prime}(t)\right| \Delta ;\right. \\
& \left.t \in K, y \in\left\langle y_{L}^{0}(t), y_{R}^{0}(t)\right\rangle, \gamma \in\langle 0,2 \pi\rangle\right]>0, \\
& \mu_{2}\left(K, \epsilon_{0}\right) \quad \\
& \leq \max \left[\frac{1}{a^{2}(t)} \sin ^{2} \gamma+\bar{f}(t, y) \cos ^{2} \gamma+\epsilon_{0}\left|u_{2}^{\prime}(t)\right| \Delta ;\right. \\
& \left.t \in K, y \in\left\langle y_{L}^{0}(t), y_{R}^{0}(t)\right\rangle, \gamma \in\langle 0,2 \pi\rangle\right]>0
\end{aligned}
$$

for sufficiently small value of upper bound $\epsilon_{0}$ of singular perturbation parameter $\epsilon$.

Further, if $\epsilon_{0}^{(1)}<\epsilon_{0}^{(2)}$, then

$$
\mu_{1}\left(K, \epsilon_{0}^{(1)}\right)>\mu_{1}\left(K, \epsilon_{0}^{(2)}\right),
$$

and conversely

$$
\mu_{2}\left(K, \epsilon_{0}^{(1)}\right)<\mu_{2}\left(K, \epsilon_{0}^{(2)}\right) .
$$

Thus we have the inequality

$$
\mu_{1}\left(K, \epsilon_{0}\right) \epsilon^{-1} \leq \gamma^{\prime} \leq \mu_{2}\left(K, \epsilon_{0}\right) \epsilon^{-1}
$$


Integrating this inequality with respect to the variable $t$ between two successive zeros $(j$ th and $(j+1)$ th, $j=1,2, \ldots)$ of $y^{\epsilon}(t)-u_{2}(t), t \in K$, we obtain immediately the lower and upper bound of their spacing $s_{\epsilon}$. Indeed,

$$
\begin{aligned}
& \int_{\text {zero }(j) \text { th }}^{\text {zero }(j+1) \text { th }} \frac{\mu_{2}\left(K, \epsilon_{0}\right)}{\epsilon} d t \geq \int_{\text {zero }(j) \text { th }}^{\text {zero }(j+1) \text { th }} \gamma^{\prime} d t \\
& \geq \int_{\text {zero }(j) \text { th }}^{\text {zero }(j+1) \text { th }} \frac{\mu_{1}\left(K, \epsilon_{0}\right)}{\epsilon} d t \\
& \frac{\mu_{2}\left(K, \epsilon_{0}\right)}{\epsilon} s_{\epsilon} \geq \pi \geq \frac{\mu_{1}\left(K, \epsilon_{0}\right)}{\epsilon} s_{\epsilon} .
\end{aligned}
$$

Hence,

$$
\epsilon \frac{\pi}{\mu_{2}\left(K, \epsilon_{0}\right)} \leq s_{\epsilon} \leq \epsilon \frac{\pi}{\mu_{1}\left(K, \epsilon_{0}\right)}, \quad \epsilon \in\left(0, \epsilon_{0}\right] .
$$

\section{Conclusion}

The frequency of nonlinear oscillations of Duffing's type equations arising via saddle-center bifurcation in associated system may be controlled by the singular perturbation parameter $\epsilon$. These oscillations are very sensitive on the initial conditions.

\section{References}

[1] Z. Feng, G. Chen, and S.-B. Hsu, "A qualitative study of the damped Duffing equation and applications," Discrete and Continuous Dynamical Systems B, vol. 6, no. 5, pp. 1097-1112, 2006.

[2] A. Litvak-Hinenzon and V. Rom-Kedar, "Symmetry-breaking perturbations and strange attractors," Physical Review E, vol. 55, no. 5, pp. 4964-4978, 1997.

[3] V. Ryabov and K. Fukushima, "Analysis of homoclinic bifurcation in Duffing oscillator under two-frequency excitation: peculiarity of using Melnikov method in combination with averaging technique," Proceedings of the Chaotic Modeling \& Simulation International Conference (CHaos '10), 2010.

[4] X. Wei, M. F. Randrianandrasana, M. Ward, and D. Lowe, "Nonlinear dynamics of a periodically driven Duffing resonator coupled to a Van der Pol oscillator," Mathematical Problems in Engineering, Article ID 248328, 16 pages, 2011.

[5] X. Yue, W. Xu, and Y. Zhang, "Global bifurcation analysis of Rayleigh-Duffing oscillator through the composite cell coordinate system method," Nonlinear Dynamics, vol. 69, no. 1-2, pp. 437-457, 2012.

[6] A. H. Nayfeh and D. T. Mook, Nonlinear Oscillations, Pure and Applied Mathematics, Wiley-Interscience, New York, NY, USA, 1979.

[7] S. Lenci and G. Rega, "Optimal control of nonregular dynamics in a Duffing oscillator," Nonlinear Dynamics, vol. 33, no. 1, pp. 71-86, 2003.

[8] A. Sharma, V. Patidar, G. Purohit, and K. K. Sud, "Effects on the bifurcation and chaos in forced Duffing oscillator due to nonlinear damping," Communications in Nonlinear Science and Numerical Simulation, vol. 17, no. 6, pp. 2254-2269, 2012.
[9] E. Tamaseviciute, A. Tamasevicius, G. Mykolaitis, S. Bumeliene, and E. Lind-berg, "Analogue electrical circuit for simulation of the duffing-holmes equation," Nonlinear Analysis: Modelling and Control, vol. 13, no. 2, pp. 241-252, 2008.

[10] V. Sauli, "Bethe-Salpeter study of radially excited vector quarkonia," Physical Review D, vol. 86, Article ID 096004, 6 pages, 2012.

[11] V. K. Dolmatov, J. L. King, and J. C. Oglesby, "Diffuse versus square-well confining potentials in modelling A@C60 atoms," Journal of Physics B, vol. 45, Article ID 105102, 2012.

[12] C. K. R. T. Jones, "Geometric singular perturbation theory," in C.I.M.E. Lectures, Montecatini Terme, vol. 1609 of Lecture Notes in Mathematics, pp. 44-118, Springer, Heidelberg, Germany, 1995.

[13] R. Vrabel and M. Abas, "Frequency control of singularly perturbed forced Duffing's oscillator," Journal of Dynamical and Control Systems, vol. 17, no. 3, pp. 451-467, 2011.

[14] S.-N. Chow, C. Z. Li, and D. Wang, Normal Forms and Bifurcation of Planar Vector Fields, Cambridge University Press, Cambridge, UK, 1994.

[15] D. Xiao and S. Ruan, "Bogdanov-Takens bifurcations in predator-prey systems with constant rate harvesting," Fields Institute Communications, vol. 21, pp. 493-506, 1999.

[16] V. Gelfreich, "Splitting of a small separatrix loop near the saddle-center bifurcation in area-preserving maps," Physica $D$, vol. 136, no. 3-4, pp. 266-279, 2000. 


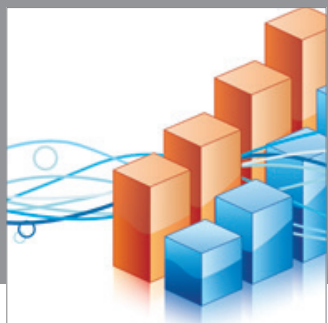

Advances in

Operations Research

mansans

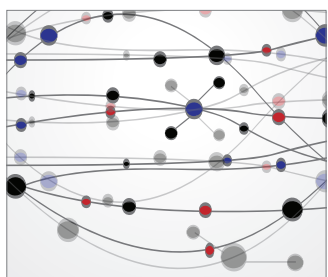

The Scientific World Journal
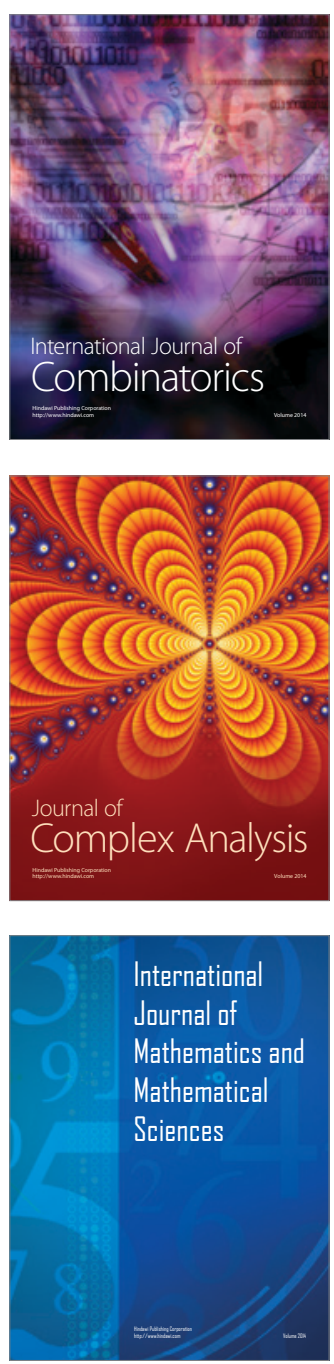
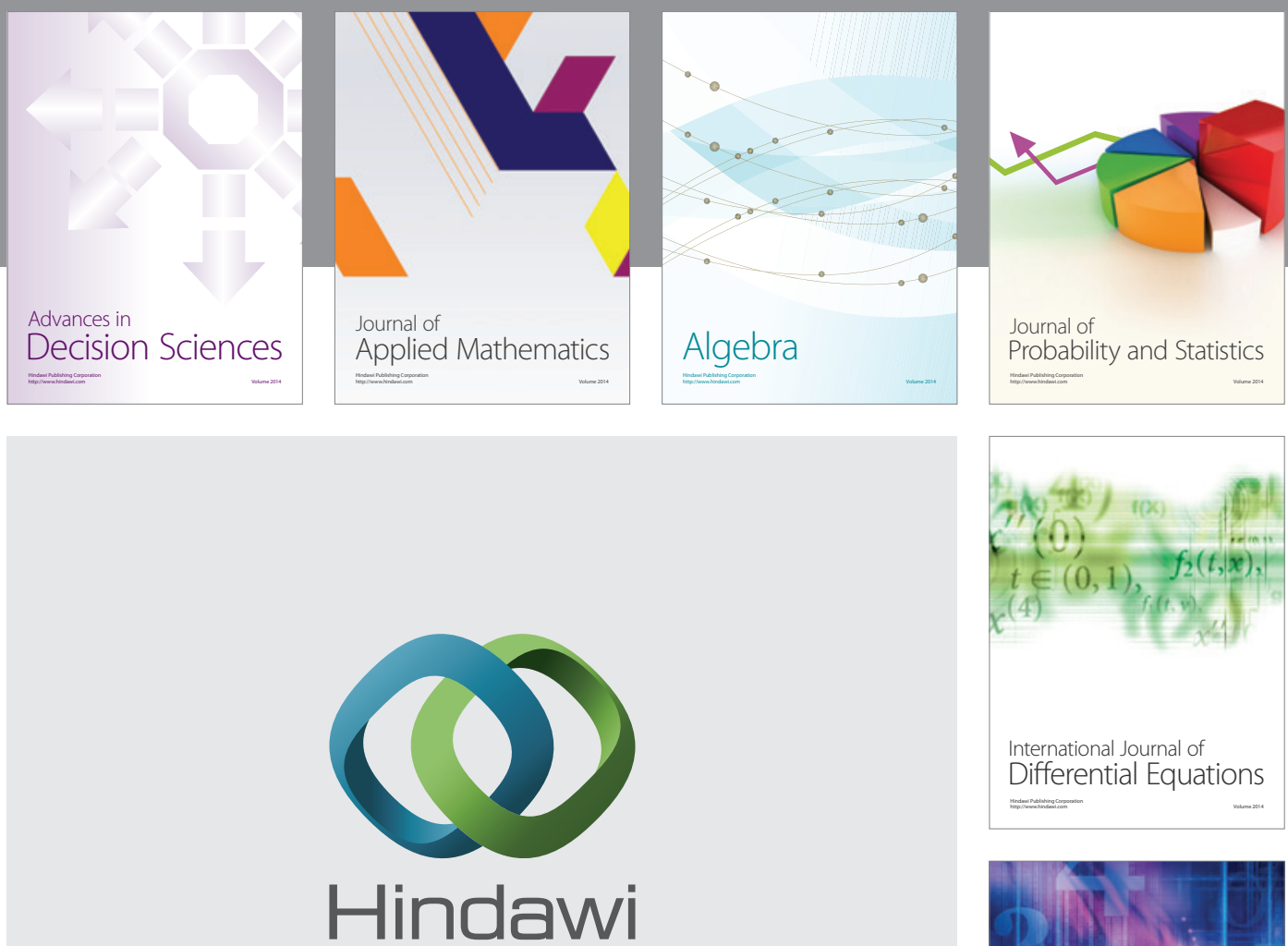

Submit your manuscripts at http://www.hindawi.com
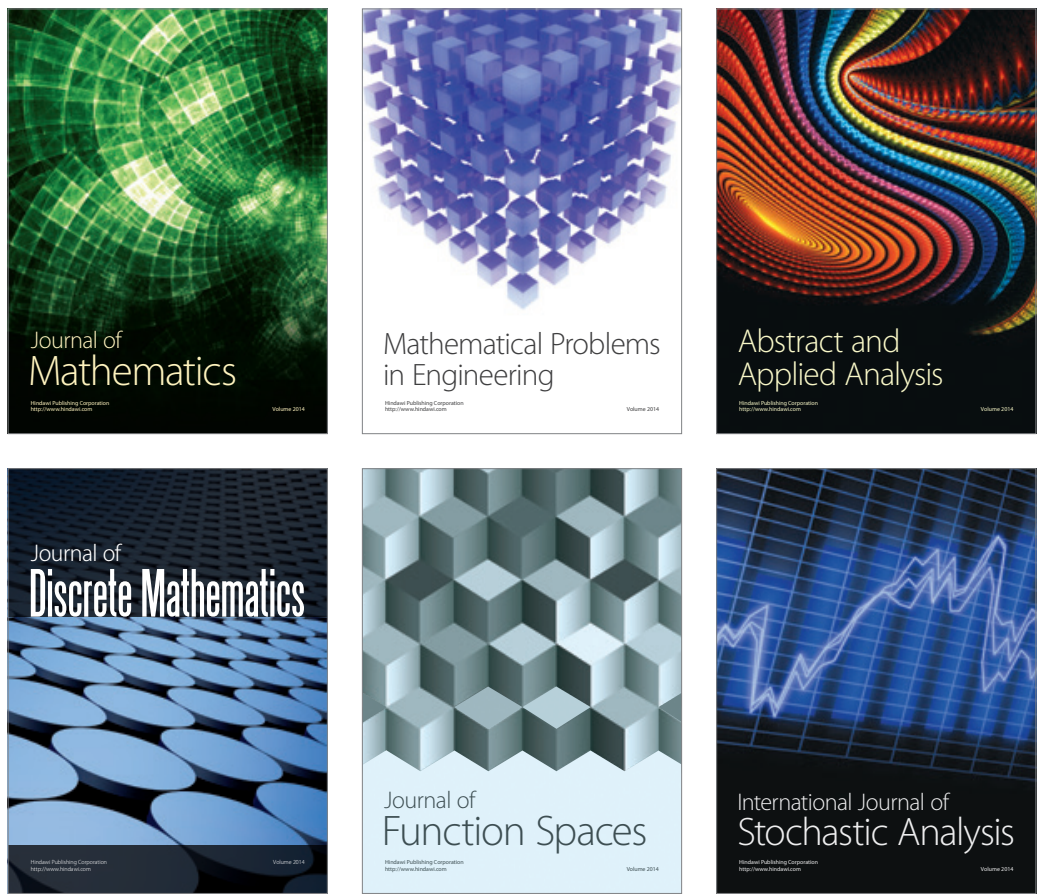

Journal of

Function Spaces

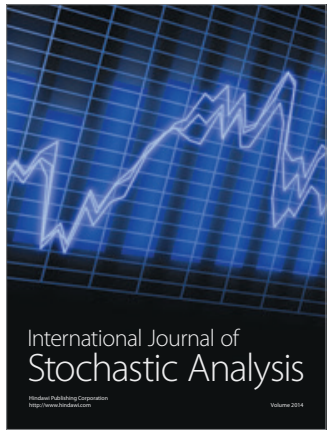

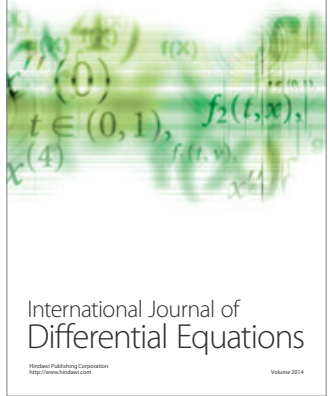
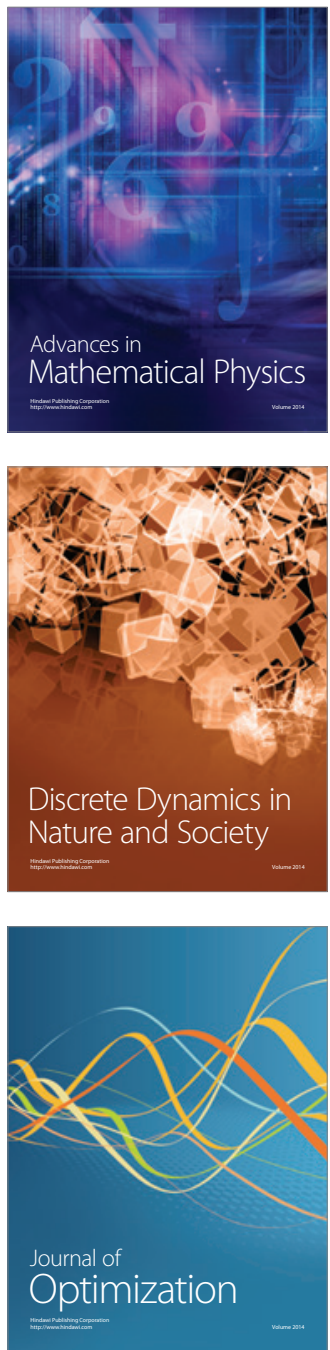